\title{
Symptomatic relief from at-home use of activated Bifidobacterium infantis EVC001 probiotic in infants: results from a consumer survey on the effects on diaper rash, colic symptoms, and sleep
}

\author{
S.M. Dimitratos ${ }^{1 *}$, H. Brown ${ }^{2}$, T. Shafizadeh ${ }^{2}$, S. Kazi ${ }^{2}$, T. Altmann ${ }^{3,4}$ and B. Ostrer ${ }^{2}$ \\ ${ }^{1}$ Department of Nutrition, University of California, Davis, Davis, CA 95616, USA ${ }^{2}$ Evolve BioSystems, Inc, Davis, CA 95618, \\ USA $;{ }^{3}$ Children's Hospital Los Angeles, Los Angeles, CA 90027, USA; ${ }^{4}$ Calabasas Pediatrics Wellness Center, Calabasas, \\ CA 91302, USA; sdimitratos@evolvebiosystems.com
}

Received: 16 December 2020 / Accepted: 28 February 2021

(c) 2021 Wageningen Academic Publishers

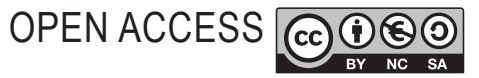

RESEARCH ARTICLE

\begin{abstract}
The gut microbiome during infancy is directly involved in the digestion of human milk, development of the immune system, and long-term health outcomes. Gut dysbiosis in early life has been linked to multiple short-term ailments, from diaper dermatitis and poor stooling habits, to poor sleep and fussiness, with mixed results in the scientific literature on the efficacy of probiotics for symptom resolution. Despite the growing interest in probiotics for consumer use, observed symptomatic relief is rarely documented. This study aims to evaluate observed symptomatic relief from at-home use of activated Bifidobacterium infantis EVC001 in infants. Consumer feedback was collected over a 2-year period via a 30-day post-purchase online survey of B. infantis EVC001 (Evivo ${ }^{\circ}$ ) customers. Outcome measures included observed changes in diaper rash, symptoms of colic, and sleep behaviours in infants fed B. infantis EVC001. A total of 1,621 respondents completed the survey. Before purchasing B. infantis EVC001, the majority of respondents visited the product website, researched infant probiotics online, or consulted with their doctor or other healthcare professional. Of the participants whose infants had ever experienced diaper rash, $72 \%(\mathrm{n}=448)$ reported improvements, and $57 \%$ of those reported complete resolution of this problem. Of those who responded to questions about gassiness/fussiness, naptime sleep, and night-time sleep behaviours, 63\% ( $n=984), 33 \%(n=520)$, and $52 \%(\mathrm{n}=806)$ reported resolution or improvements, respectively. Although clinical data regarding probiotic use are often inconclusive for symptom resolution, home use of B. infantis EVC001 in infants improved diaper rash, gassiness/fussiness, and sleep quality within the first week of use in a significant number of respondents who engaged in a voluntary post-purchase survey. These outcomes may be a result of the unique genetic capacity of B. infantis EVC001 to colonise the infant gut highlighting the importance of strain selection in evaluating the effects of probiotic products.
\end{abstract}

Keywords: host-microbe interactions, B. infantis, infant gut microbiome, probiotics

\section{Introduction}

The gut microbiome is inextricably tied to human health and disease. Studies, primarily in adults, have demonstrated a role for the gut microbiome in inflammatory bowel disease (Nishida et al., 2018), mood disorders (Evrensel and Ceylan, 2015), autoimmunity (Brown et al., 2019), obesity (TorresFuentes et al., 2017), and metabolic disorders such as diabetes (He et al., 2015). New data show a particularly important role for the gut microbiome during infancy. Diaper rash, colic, and sleep problems in infancy are common concerns for parents, and emerging evidence strongly suggest a role for host-microbiome interactions underlying these common conditions that affect infants. For example, common maladies such as colic have been associated with intestinal inflammation and dysbiosis (Rhoads et al., 2018). Gut dysbiosis is described as a low-functioning microbiome with high susceptibility to invasion by pathogenic bacteria, 
which are linked to negative health outcomes. Furthermore, dysbiosis is the absence of specific bacteria (Bifidobacterium infantis in the case of the infant gut) known to provide essential function to the gut (Duar et al., 2020a). Additionally, diaper dermatitis has been linked to elevated faecal $\mathrm{pH}$, which is also tied to certain changes in the infant gut microbiome (Berg et al., 1994). Emerging evidence provides a strong case for addressing infant gut dysbiosis as a remedy for diaper rash, colic, sleep problems, among other maladies, but published data on efficacy is inconclusive.

Reports of infants with diaper dermatitis (diaper rash) are widespread, with literature estimates ranging from 16-65\% (Carr et al., 2020). Solutions have traditionally included prophylactic ointment or cream containing zinc oxide, frequent diaper changes, and frequent bathing (Carr et al., 2020). Colic has been reported to affect between $17-25 \%$ of infants (Wolke et al., 2017). Colic is clinically characterised by inconsolable crying for $>3 \mathrm{~h},>3$ days a week, for $>3$ weeks and its resolution has included dietary modifications, such as use of hydrolysed formulas, lactase enzymes, low allergen maternal diets, and low-allergen formulas; however, data on the efficacy of these methods are inconclusive, and none of these dietary modifications are currently recommended (Gordon et al., 2018). Further options for colic have included parental education and reassurance, behavioural modifications including parent coping behaviours, swaddling, baby massage, carrying the child, use of sucrose, simethicone, and lactase, use of probiotics, and use of alternative interventions such as herbal remedies, acupuncture, manipulative therapy, and reflexology (Zeevenhooven et al., 2018). Results from research on these remedies vary, and there are currently no standard recommendations (Zeevenhooven et al., 2018). Infantile sleep problems (e.g. trouble falling or staying asleep) are other common concern for parents of infants, and sleep problems have been associated with adverse consequences including parental depression, fatigue, poor health, and psychological distress (Reuter et al., 2020). Sleep problems have been reported to affect between 1535\% of infants (Field, 2017; Reuter et al., 2020); however, estimates vary widely in the literature due, in part, to inconsistencies in defining 'sleep problems.' Options for parents have included such things as consultations by a health professional, parental education on extinction and bedtime fading, internet-based interventions, and nighttime massage (Field, 2017). Taken together, traditional remedies for diaper rash, symptoms of colic, and sleep problems demonstrate mixed efficacy in the literature with no clear recommendations for improving these conditions.

New options for these concerns should address underlying issues of the infant gut microbiome, and a growing body of literature suggests a promising role for Bifidobacterium longum subspecies infantis (B. infantis) in promoting infant gut health. $B$. infantis is a gram-positive intestinal symbiont that historically dominated the infant gastrointestinal tract (Henrick et al., 2018). Studies have found that this bacterium confers multiple known benefits to infants (Underwood et al., 2015). Despite the known benefits of $B$. infantis, there has been a profound reduction in total Bifidobacterium in the infant gut over the past century (Henrick et al., 2018; Tannock et al., 2016). It is unclear whether this is the product of environmental and/or lifestyle factors, and perhaps in combination with interventions such as caesarean sections (C-sections), antibiotic use (Yassour et al., 2016), and formula feeding, all of which have been great advancements to human health but have also had deleterious, unintended side effects on the infant gut microbiome.

The infant gut requires infant-specific bacteria to metabolise human milk oligosaccharides (HMOs) from human milk. $B$. infantis produces lactate and acetate as acidic fermentation products from HMO metabolism, contributing, at least in part, to a reduction in faecal $\mathrm{pH}$ and an increase in colonisation resistance (Casaburi and Frese, 2018). Acetate, a short chain fatty acid (SCFA), serves numerous protective roles in the infant gut (Fukuda et al., 2011). Studies reporting on probiotic efficacy against necrotising enterocolitis (NEC), the most common and potentially fatal gastrointestinal disease that primarily affects preterm infants, have been mixed (Suez et al., 2019). Due to the lack of studies in general, consumer studies have documented mixed interest about using probiotics for small children. For example, one Danish study found that parents were sceptical about the use of probiotics for disease prevention in their children (Andersen et al., 2018). Another study found that the majority of mothers in a birth cohort believed that probiotics in general were beneficial, however, there remained uncertainty regarding the safety and therapeutic implications for infants (Bridgman et al., 2014). These data warrant further studies to clearly demonstrate symptomatic relief and safety of infant probiotics.

In addition to its availability for hospital use, $B$. infantis EVC001 is also available for use at home, and consumer evaluations of this probiotic have been carefully monitored for over two years. Given highlighted gaps in the literature around consumer observed symptomatic relief with probiotic administration in young children, our goal for this research was to examine consumer observations of feeding $B$. infantis EVC001 to their infants. Through use of an online, post-purchase voluntary survey, we queried consumers regarding symptomatic relief from feeding this strain of probiotic to their infants at home, specifically focusing on changes in diaper rash, sleep patterns, and colic symptoms. We hypothesised that the majority of parents who purchased B. infantis EVC001 (1) were motivated to purchase the product based on information gathered from internet searches and from 
recommendations from healthcare providers, friends and family, and (2) would observe improvements in symptoms of colic, diaper rash and sleep behaviours in their infants after using B. infantis EV001.

\section{Materials and methods}

\section{Study subjects and survey administration}

Participants were recruited via email from a pool of consumers who had purchased activated B. infantis EVC001 (Evivo ${ }^{\circ}$; Evolve BioSystems, Inc., Davis, CA, USA) in the previous 30-day period. These consumers were asked to complete a voluntary survey regarding their experience and observations from use of $B$. infantis EVC001 with their infant $(\mathrm{n}=1,621)$ The survey was administered electronically via Survey Monkey from March $20^{\text {th }}, 2018$ to June $15^{\text {th }}$, 2020. Participants who completed the survey were provided with the opportunity to enter into a prize drawing to win a \$50 Amazon.com gift card. Supplementary Table S1 describes the survey questions in more detail.

\section{Ethical considerations}

A central IRB (Sterling IRB, registration number IRB00001790) reviewed the study protocol and determined that the research was exempt from IRB approval because the survey and associated data collection did not meet the criteria for human subject research. Participation in the survey was completely voluntary.

\section{Data analysis}

Survey questions regarding perceived changes in diaper rash, fussiness/gassiness, and sleep were compiled and responses were binned into 'improvement', 'no change' and 'worse' categories. Frequencies of categories, as well as time to perceived changes, were calculated. Chi-squared tests for equality of proportions were performed for perceived change response rates. Time to perceived change responses of 'immediately', 'within a week' and 'within two weeks' were combined, and proportion of grouped responses was calculated.

\section{Diaper rash}

When asked if they noticed any differences relating to diaper rash, respondents selected one of the following: 'no longer has diaper rash', 'less diaper rash', 'about the same amount of diaper rash', 'more diaper rash', or 'my baby has never had diaper rash'. Participants who marked that their baby has never had diaper rash were omitted from the analysis of changes in diaper rash. Responses of 'no longer has diaper rash' and 'less diaper rash' were considered improvement and were combined for the purposes of analysis. The responses of 'about the same amount of diaper rash' and 'more diaper rash' were considered as 'no change' and 'worse', respectively.

\section{Gassiness and fussiness}

Consumers were asked whether their baby's fussiness/ gassiness was 'less', 'about the same', or 'more' after feeding B. infantis EVC001 to their infant. Responses of 'less' were considered as improvement, 'about the same' as no change, and 'more' as worse in perceived symptomatic change.

\section{Sleep behaviour}

Change in night-time sleep and napping patterns were described as one of the following: 'longer duration', 'more consistent', 'longer duration and more consistent,' 'sleeps about the same,' 'shorter duration,' 'less consistent,' 'shorter duration and less consistent'. Responses including longer or more consistent duration were considered as improvement in perceived change. 'Sleeps about the same' was considered as no change, and shorter or less consistent responses were considered as worse in perceived change.

Overall, respondents who reported seeing improvement in any of the observed symptoms were further asked whether those changes were observed 'immediately', 'within the first week,' 'within two weeks', or 'after about a month'.

\section{Results}

\section{Demographics}

Between March $20^{\text {th }}, 2018$ and June $15^{\text {th }}, 2020,15,172$ individuals who purchased $B$. infantis EVC001 were invited by email to participate in the survey within 30 days post-purchase. A total of 1,621 questionnaires were completed, representing a response rate of $10.7 \%$. Data were not collected from those who declined to be involved. Respondent characteristics are presented in Table 1.

\section{Age of baby when first hearing about Bifidobacterium infantis EVCO01}

A total of 1,192 respondents provided information about the age of their baby when they first heard about $B$. infantis EVC001. Nearly three-quarters $(71 \%, \mathrm{n}=846)$ of respondents first heard about this product early in their baby's life (1-8 weeks old), with $22 \%$ ( $n=263$ ) having a baby $1-2$ weeks old, and $49 \%(n=583)$ having a baby 3-8 weeks old.

\section{Prevalence of pre-purchase research on baby probiotics}

A large number of respondents performed research on their own or inquired of healthcare professionals before making their purchase of B. infantis EVC001. A total of 1,353 participants provided their perspective on what 
Table 1. Respondent purchase behaviour.

\begin{tabular}{|c|c|c|}
\hline Response & $\mathrm{n}(\%)$ & Total respondents \\
\hline \multicolumn{3}{|l|}{ How old was your baby when you first heard about Evivo? } \\
\hline $1-2$ weeks & $263(22 \%)$ & 1,192 \\
\hline 3-8 weeks & $583(49 \%)$ & \\
\hline 2-3 months & $173(15 \%)$ & \\
\hline 3 months or older & $173(15 \%)$ & \\
\hline \multicolumn{3}{|c|}{ Did you do any of the following between the time you first heard about } \\
\hline \multicolumn{3}{|c|}{ Evivo and the time you purchased? (select all that apply) ${ }^{1}$} \\
\hline Consulted with my doctor/healthcare professional & $591(44 \%)$ & 1,353 \\
\hline Researched infant probiotics online & $1,017(75 \%)$ & \\
\hline Read through the Evivo website & $1,215(90 \%)$ & \\
\hline Talked to friends/family to get their opinion & $409(30 \%)$ & \\
\hline Other & $101(7 \%)$ & \\
\hline
\end{tabular}

actions they may have taken between visiting Evivo.com and making their purchase. $90 \%(n=1,215)$ read through the Evivo website, $75 \%(n=1,017)$ researched probiotics online, and $44 \%(n=591)$ consulted with their doctor or healthcare professional before making their purchase.

\section{Changes in infant diaper rash}

A total of 1,548 respondents answered questions regarding symptoms of diaper rash observed in their infant. Diaper rash is a problem experienced by a large number of infants included in this survey, with over $40 \%(n=622)$ of respondents reporting some experience with the problem (Figure 1). After feeding B. infantis EVC001, 72\% ( $\mathrm{n}=448)$ of those that had experienced diaper rash observed improvements in diaper rash in their baby. Of those that observed improvement, the largest group $(57 \%, \mathrm{n}=255)$ no longer experienced diaper rash, with $43 \%(n=193)$ reporting having less diaper rash. Of those that experienced an improvement, nearly all $(96 \%, n=428)$ noticed changes in diaper rash either immediately $(21 \%, n=93)$, within the first week ( $53 \%, \mathrm{n}=236)$ or within two weeks $(22 \%, \mathrm{n}=99)$ (Figure 2). The remainder observed improvement after a month $(4 \%, n=20)$. A 3 way chi-squared test for equality of proportions revealed that the response rates among improved, no change and worse responses were significantly different $(P<0.0001)$.

\section{Changes in gassiness and fussiness}

A total of 1,553 respondents answered questions regarding observed gassiness and fussiness, both of which are considered symptoms of colic. After feeding B. infantis
EVC001, nearly two-thirds of participants $(63 \%, \mathrm{n}=984)$ observed improvements in gassiness and fussiness (Figure 1), and response rates among improvement, no change and worse response categories was found to be significantly different $(P<0.0001)$. Not only did most see improvement in these symptoms of colic, but $94 \%(n=924)$ of participants observed these improvements quickly after feeding B. infantis EVC001 with 31\% $(\mathrm{n}=306)$ reporting change within two weeks, nearly half $(49 \%, n=481)$ seeing change within the first week, and $14 \%(n=137)$ seeing immediate change in their baby's gassiness and fussiness (Figure 2). The remainder saw improvement after about a month $(6 \%, \mathrm{n}=58)$.

\section{Changes in night-time sleep}

A total of 1,556 respondents answered questions regarding night-time sleep behaviour. Of the total respondents, over half $(52 \%, \mathrm{n}=806)$ reported improvement in night-time sleep after feeding $B$. infantis EVC001, with the majority $(30 \%, n=460)$ seeing improvement in both duration and consistency of night-time sleep (Figure 1). 9\% $(n=136)$ observed more consistent sleep, and 13\% $(n=210)$ reported longer duration of night-time sleep. The response rates of improved, no change and worse categories were found to be significantly different $(P<0.0001)$. The observed improvements in night-time sleep occurred quickly after feeding B. infantis EVC001, with most $(86 \%, \mathrm{n}=692)$ seeing improvements in night-time sleep either immediately ( $9 \%$, $\mathrm{n}=69)$, within the first week $(41 \%, \mathrm{n}=330)$ or within two weeks (36\%, n=292) (Figure 2). The remainder observed improvements after about a month $(14 \%, \mathrm{n}=113)$. 


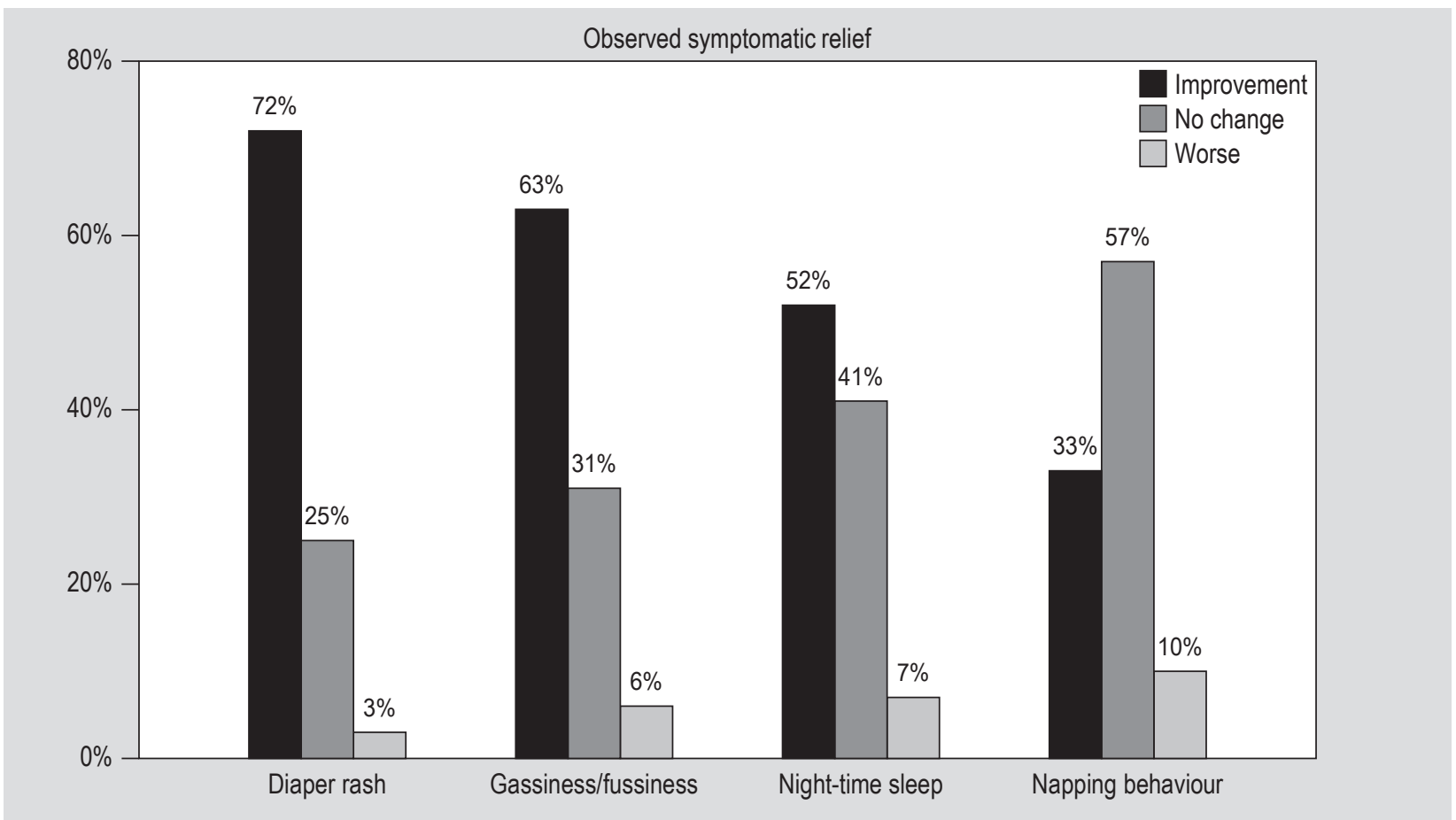

Figure 1. Percentage of survey respondents who reported either improvement, no change or worse symptoms in their infants following the consumption of Bifidobacterium infantis EVC001.

\section{Changes in napping behaviour}

A total of 1,565 respondents answered questions regarding napping behaviour of their infant. Response rates among improvement, no change and worse categories were found to be significantly different $(P<0.0001)$. One-third $(33 \%$, $\mathrm{n}=520)$ reported an improvement in napping duration, consistency, or a combination of the two, with the majority $(16 \%, n=252)$ seeing improvement in both duration and consistency of napping behaviour after feeding $B$. infantis EVC001 (Figure 1). 7\% ( $\mathrm{n}=113)$ noted improvement in only the duration of napping, and 10\% ( $\mathrm{n}=155)$ observed more consistent napping. The observed improvements in napping behaviour occurred quickly after feeding B. infantis EVC001, with 90\% ( $\mathrm{n}=474)$ seeing napping improvements either immediately $(11 \%, \mathrm{n}=58)$, within the first week of feeding B. infantis EVC001 (44\%, $\mathrm{n}=226)$ or within two weeks (36\%, $\mathrm{n}=187)$ (Figure 2$)$. The remainder observed improvements after about a month $(9 \%, \mathrm{n}=47)$.

\section{Discussion}

\section{Bifidobacterium infantis colonisation creates a protective environment in the infant gut}

Exclusively breastfed infants receive an abundance of diverse glycans from human milk, a significant portion of which are HMOs which serve as the primary nutrition source for the infant's early-life gut bacteria (Duar et al.,

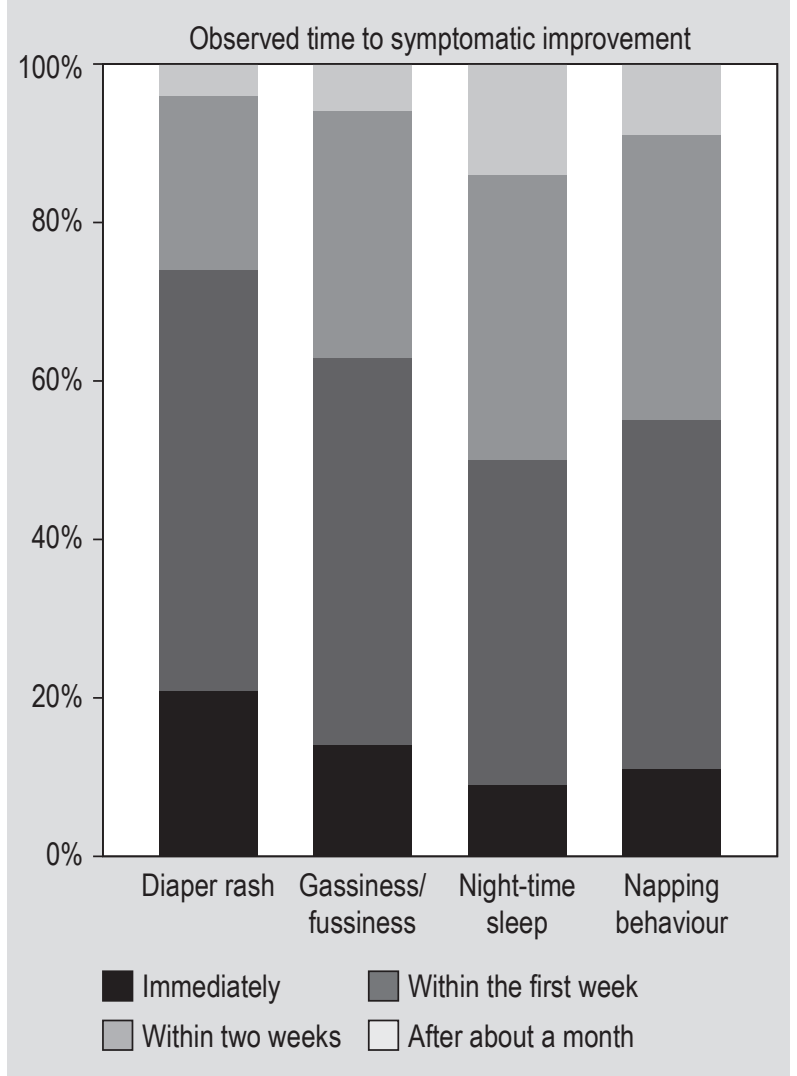

Figure 2. Percentage of survey respondents reporting observing symptom improvement within the four categories provided (immediately, within the first week, within two weeks and after about a month). 
2020a; Smilowitz et al., 2014). Interestingly, humans lack enzymes for the digestion of HMOs, but these glycans are known to provide a medium that selectively promotes the growth of $B$. infantis, a gut symbiont that completely utilises HMOs to produce important organic acid by-products, such as lactate and acetate. These milk glycans serve many functions: promoting development of the immune system, binding viruses, enhancing epithelial barrier function, and selectively enriching $B$. infantis in the infant gut (Smilowitz et al., 2014). It has been demonstrated that $B$. infantis EVC001 prodigiously consumes HMOs and creates a protective environment in the developing neonatal gut (Casaburi and Frese, 2018; Duar et al., 2020b; Henrick et al., 2019; Karav et al., 2018).

Colonisation resistance refers to a process by which the invasion and overgrowth of pathogenic bacteria is prevented (Duar et al., 2020b; Lawley and Walker, 2013). B. infantis facilitates the process of colonisation resistance, thereby creating a protective environment in the infant gut (Duar et al., 2020b). Infants in primarily industrialised parts of the world (e.g. the United States and Europe) are believed to now have reduced colonisation resistance due to the unintended consequences of important and often life-saving practices, including infant formula use, C-section births, and maternal and infant use of antibiotics, resulting in less abundant (or absent) B. infantis in the infant gastrointestinal tract. Previous studies have shown that breastfed infants colonised with B. infantis EVC001, had reduced excretion of HMOs in their stool, higher faecal lactate and acetate, and therefore lower faecal $\mathrm{pH}$ compared to control infants (Frese et al., 2017). This natural mechanism by which $B$. infantis lowers colonic and faecal $\mathrm{pH}$ through the production of lactate and acetate inhibits the growth of potentially pathogenic bacteria and reduces infection risk in the infant gut (Fukuda et al., 2011).

In this study, we found that $72 \%$ of respondents reported symptomatic relief in diaper rash, $63 \%$ in gassiness and fussiness, $52 \%$ in night-time sleep, and $33 \%$ in napping behaviours after giving $B$. infantis EVC001 to their infant at home (Figure 1). Not only did the majority of respondents report relief in these three domains, but the time to relief was remarkable as well (Figure 2). The mechanism of action of B. infantis EVC001 colonisation in the infant gut has been previously identified, and is based largely on the unique genetic capability of this strain to completely metabolise HMOs found in human milk into lactate and acetate, acidic end products that lead to lower colonic and faecal $\mathrm{pH}$ compared to infants missing this bacterium (Frese et al., 2017). Furthermore, the time course of the colonisation of B. infantis EVC001 in the gut of the breastfed infant has been shown to occur within one week (Frese et al., 2017), a timeframe consistent with the resolution of the study's symptoms in most cases.
In the absence of HMO-metabolising bacteria, namely B. infantis, bacterial production of lactate and acetate is limited, resulting in higher colonic and faecal $\mathrm{pH}$ (Frese et al., 2017). As faecal enzymes are shown to be activated at $\mathrm{pH}$ levels above 5.5, the absence of B. infantis in the infant gut may contribute to increased faecal enzyme activity and skin barrier breakdown, resulting in increased incidence of diaper rash (Berg et al., 1994). In this study, $72 \%$ of surveyed customers whose baby had ever experienced diaper rash reported a reduction in diaper rash with use of $B$. infantis EVC001 and in 74\% of those infants, the diaper rash was resolved within one week.

The presence of $B$. infantis in the infant gut facilitates colonisation resistance (Duar et al., 2020b), thereby reducing pathogens linked to gas production, inflammation (Henrick et al., 2019), and colic (Rhoads et al., 2018). In a previous study of $B$. infantis EVC001, colonisation of the infant gut occurred rapidly (within the first week) and displaced a significant proportion of the potentially pathogenic bacteria that were previously identified in faecal samples from the infants (Frese et al., 2017). This mechanism may explain not only why $63 \%$ of respondents reported symptomatic relief in gassiness and fussiness in their infants after feeding B. infantis EVC001, but also why $63 \%$ of those who reported improvement, saw improvements within the first week.

Of the $52 \%$ of respondents who reported improved night-time sleep in their infants, 50\% saw improvement immediately or within the first week (Figure 2). Additionally, of the $33 \%$ of respondents who reported improved naptime behaviours, 55\% saw improvements within the first week. These observed benefits may be attributable to the improved metabolism and utilisation of HMOs, which are otherwise excreted through stool in infants missing B. infantis. Also, B. infantis EVC001 has been shown to reduce gastrointestinal inflammation in infants, and therefore may explain the improved sleep behaviour as a result of less discomfort and fussiness.

\section{Strengths and limitations}

This study aimed to evaluate observed symptomatic relief with at-home use of B. infantis EVC001 in infants. This study had many strengths, including a large sample size (>1,600 participants), a consumer-friendly survey format, and multiple outcome measures (e.g. diaper rash, gassiness/ fussiness, naptime sleep behaviour, and night-time sleep behaviour. Additionally, the survey documented and disclosed when respondents reported 'no change' and/or worsening of symptoms in response to product use.

This study does not come without limitations. For example, our study could not correct for infant age, infant diet, and other existing health and/or medical conditions in the infants receiving $B$. infantis EVC001. Our study did 
not have a control group (those receiving no B. infantis EVC001), thus future work should address this limitation to determine definitive efficacy. Another limitation pertains to possible bias (for example, recall bias or responder bias). With regard to survey completion specifically, it is possible that respondents who had very positive or very negative experiences could be more likely to participate. Additionally, we reported on symptoms of colic including parental reporting of infant fussiness at home, rather than colic as it is defined clinically (inconsolable crying for $>3 \mathrm{~h}$ a day, $>3$ days a week, for $>3$ weeks). Finally, the effects of other remedies used in addition to $B$. infantis EVC001 were not captured in this survey. Future consumer research should address these limitations in the study design.

\section{Conclusions}

Feeding activated $B$. infantis EVC001 probiotic to infants in an at-home setting resulted in observed symptomatic relief of diaper rash, gassiness/fussiness, and sleep quality in a voluntary consumer survey. We also report that most symptomatic relief occurred within the first week of use. We believe this outcome may be attributed to the specific genetic capacity of $B$. infantis EVC001 to colonise the infant gut, utilising a mechanism distinct from other probiotic bacteria, thereby highlighting the importance of strain selection of probiotic use.

\section{Supplementary material}

Supplementary material can be found online at https://doi. org/10.3920/BM2020.0229.

All data generated or analysed during this study are included in this published article and can be found in Supplementary Table S1.

Table S1. Survey questionnaire with responses.

\section{Conflict of interest}

BO, HB, SK, and TS are employed by Evolve BioSystems, Inc. SD and TA are consultants for Evolve BioSystems, Inc.

\section{Acknowledgements}

We thank Carol Maggio for assisting in the development and programming of the survey used in this study, and for her contribution to final data analysis. This study was funded by Evolve BioSystems, Inc.

\section{References}

Andersen, S.S., Michaelsen, K.F., Laursen, R.P. and Holm, L., 2018. Why parents are skeptical about using probiotics preventively for small children: a Danish qualitative study. BMC Complementary and Alternative Medicine 18: 336. https://doi.org/10.1186/s12906018-2387-2

Berg, R.W., Milligan, M.C. and Sarbaugh, F.C., 1994. Association of skin wetness and $\mathrm{pH}$ with diaper dermatitis. Pediatric Dermatology 11: 18-20. https://doi.org/10.1111/j.1525-1470.1994.tb00066.x

Bridgman, S.L., Azad, M.B., Field, C.J., Letourneau, N., Johnston, D.W., Kaplan, B.J. and Kozyrskyj, A.L., 2014. Maternal perspectives on the use of probiotics in infants: a cross-sectional survey. BMC Complementary and Alternative Medicine 14: 366. https://doi. org/10.1186/1472-6882-14-366

Brown, E.M., Kenny, D.J. and Xavier, R.J., 2019. Gut microbiota regulation of $\mathrm{T}$ cells during inflammation and autoimmunity. Annual Review of Immunology 37: 599-624. https://doi.org/10.1146/ annurev-immunol-042718-041841

Carr, A.N., DeWitt, T., Cork, M.J., Eichenfield, L.F., Fölster-Holst, R., Hohl, D., Lane, A.T., Paller, A., Pickering, L., Taieb, A., Cui, T.Y., Xu, Z.G., Wang, X., Brink, S., Niu, Y., Ogle, J., Odio, M. and Gibb, R.D., 2020. Diaper dermatitis prevalence and severity: global perspective on the impact of caregiver behavior. Pediatric Dermatology 37: 130-136. https://doi.org/10.1111/pde.14047

Casaburi, G. and Frese, S.A., 2018. Colonization of breastfed infants by Bifidobacterium longum subsp. infantis EVC001 reduces virulence gene abundance. Human Microbiome Journal 9: 7-10. https://doi. org/10.1016/j.humic.2018.05.001

Duar, R.M., Henrick, B.M., Casaburi, G. and Frese, S.A., 2020a. Integrating the ecosystem services framework to define dysbiosis of the breastfed infant gut: the role of $B$. infantis and human milk oligosaccharides. Frontiers in Nutrition 7: 33. https://doi. org/10.3389/fnut.2020.00033

Duar, R.M., Kyle, D. and Casaburi, G., 2020b. Colonization resistance in the infant gut: the role of $B$. infantis in reducing $\mathrm{pH}$ and preventing pathogen growth. High-Throughput 9: 7. https://doi.org/10.3390/ ht9020007

Evrensel, A. and Ceylan, M.E., 2015. The gut-brain axis: the missing link in depression. Clinical Psychopharmacology and Neuroscience 13: 239-244. https://doi.org/10.9758/cpn.2015.13.3.239

Field, T., 2017. Infant sleep problems and interventions: a review. Infant Behavior and Development 47: 40-53. https://doi.org/10.1016/j. infbeh.2017.02.002

Frese, S.A., Hutton, A.A., Contreras, L.N., Shaw, C.A., Palumbo, M.C., Casaburi, G., Xu, G., Davis, J.C.C., Lebrilla, C.B., Henrick, B.M., Freeman, S.L., Barile, D., German, J.B., Mills, D.A., Smilowitz, J.T. and Underwood, M.A., 2017. Persistence of supplemented Bifidobacterium longum subsp. infantis EVC001 in breastfed infants. MSphere 2: e00501-17. https://doi.org/10.1128/msphere.00501-17 Fukuda, S., Toh, H., Hase, K., Oshima, K., Nakanishi, Y., Yoshimura, K., Tobe, T., Clarke, J.M., Topping, D.L., Suzuki, T., Taylor, T.D., Itoh, K., Kikuchi, J., Morita, H., Hattori, M. and Ohno, H., 2011. Bifidobacteria can protect from enteropathogenic infection through production of acetate. Nature 469: 543-549. https://doi.org/10.1038/ nature09646 
Gordon, M., Biagioli, E., Sorrenti, M., Lingua, C., Moja, L., Banks, S.S.C., Ceratto, S. and Savino, F., 2018. Dietary modifications for infantile colic. Cochrane Database of Systematic Reviews 10: CD011029. https://doi.org/10.1002/14651858.CD011029.pub2

He, C., Shan, Y. and Song, W., 2015. Targeting gut microbiota as a possible therapy for diabetes. Nutrition Research 35: 361-367. https://doi.org/10.1016/j.nutres.2015.03.002

Henrick, B.M., Chew, S., Casaburi, G., Brown, H.K., Frese, S.A., Zhou, Y., Underwood, M.A. and Smilowitz, J.T., 2019. Colonization by B. infantis EVC001 modulates enteric inflammation in exclusively breastfed infants. Pediatric Research 86: 749-757. https://doi. org/10.1038/s41390-019-0533-2

Henrick, B.M., Hutton, A.A., Palumbo, M.C., Casaburi, G., Mitchell, R.D., Underwood, M.A., Smilowitz, J.T. and Frese, S.A., 2018. Elevated fecal $\mathrm{pH}$ indicates a profound change in the breastfed infant gut microbiome due to reduction of Bifidobacterium over the past century. MSphere 3: e00041-18. https://doi.org/10.1128/ msphere.00041-18

Karav, S., Casaburi, G. and Frese, S.A., 2018. Reduced colonic mucin degradation in breastfed infants colonized by Bifidobacterium longum subsp. infantis EVC001. FEBS Open Bio 8: 1649-1657. https://doi.org/10.1002/2211-5463.12516

Lawley, T.D. and Walker, A.W., 2013. Intestinal colonization resistance. Immunology 138: 1-11. https://doi.org/10.1111/j.13652567.2012.03616.x

Nishida, A., Inoue, R., Inatomi, O., Bamba, S., Naito, Y. and Andoh, A., 2018. Gut microbiota in the pathogenesis of inflammatory bowel disease. Clinical Journal of Gastroenterology 11: 1-10. https://doi. org/10.1007/s12328-017-0813-5

Reuter, A., Silfverdal, S., Lindblom, K. and Hjern, A., 2020. A systematic review of prevention and treatment of infant behavioural sleep problems. Acta Paediatrica 109: 1717-1732. https://doi.org/10.1111/ apa.15182

Rhoads, J.M., Collins, J., Fatheree, N.Y., Hashmi, S.S., Taylor, C.M., Luo, M., Hoang, T.K., Gleason, W.A., Van Arsdall, M.R., Navarro, F.N. and Liu, Y., 2018. Infant colic represents gut inflammation and dysbiosis. Journal of Pediatrics 203: 55-61. https://doi.org/10.1016/j. jpeds.2018.07.042
Smilowitz, J.T., Lebrilla, C.B., Mills, D.A., German, J.B. and Freeman, S.L., 2014. Breast milk oligosaccharides: structure-function relationships in the neonate. Annual Review of Nutrition 34: 143169. https://doi.org/10.1146/annurev-nutr-071813-105721

Suez, J., Zmora, N., Segal, E. and Elinav, E., 2019. The pros, cons, and many unknowns of probiotics. Nature Medicine 25: 716-729. https://doi.org/10.1038/s41591-019-0439-x

Tannock, G.W., Lee, P.S., Wong, K.H. and Lawley, B., 2016. Why don't all infants have bifidobacteria in their stool? Frontiers in Microbiology 7: 834. https://doi.org/10.3389/fmicb.2016.00834.

Torres-Fuentes, C., Schellekens, H., Dinan, T.G. and Cryan, J.F., 2017. The microbiota-gut-brain axis in obesity. The Lancet Gastroenterology and Hepatology 2: 747-756. https://doi.org/10.1016/ S2468-1253(17)30147-4

Underwood, M.A., German, J.B., Lebrilla, C.B. and Mills, D.A., 2015. Bifidobacterium longum subspecies infantis: champion colonizer of the infant gut. Pediatric Research 77: 229-235. https://doi. org/10.1038/pr.2014.156

Wolke, D., Bilgin, A. and Samara, M., 2017. Systematic review and meta-analysis: fussing and crying durations and prevalence of colic in infants. Journal of Pediatrics 185: 55-61. https://doi.org/10.1016/j. jpeds.2017.02.020

Yassour, M., Vatanen, T., Siljander, H., Hämäläinen, A.M., Härkönen, T., Ryhänen, S.J., Franzosa, E.A., Vlamakis, H., Huttenhower, C., Gevers, D., Lander, E.S., Knip, M., the DIABIMMUNE Study Group and Xavier, R.J., 2016. Natural history of the infant gut microbiome and impact of antibiotic treatment on bacterial strain diversity and stability. Science Translational Medicine 8: 343ra81. https://doi. org/10.1126/scitranslmed.aad0917

Zeevenhooven, J., Browne, P.D., L'Hoir, M.P., De Weerth, C. and Benninga, M.A., 2018. Infant colic: mechanisms and management. Nature Reviews Gastroenterology and Hepatology 15: 479-496. https://doi.org/10.1038/s41575-018-0008-7 